\title{
A Case of Inflammatory Generalized Type of Peeling Skin Syndrome Possibly Caused by a Homozygous Missense Mutation of CDSN
}

\author{
Hiroshi Kawakami ${ }^{a}$ Masaki Uchiyama ${ }^{a}$ Tatsuo Maeda ${ }^{a}$ \\ Takahiko Tsunoda $^{b}$ Yoshihiko Mitsuhashi ${ }^{a}$ Ryoji Tsuboi ${ }^{\mathrm{a}}$ \\ ${ }^{a}$ Department of Dermatology, Tokyo Medical University, Tokyo, and \\ ${ }^{b}$ Department of Dermatology, Yamagata City Hospital Saiseikan, Yamagata, Japan
}

\section{Key Words}

Inflammatory generalized peeling skin syndrome $\cdot C D S N \cdot$ Corneodesmosomes .

Homozygous missense mutation

\begin{abstract}
A 54-year-old Japanese woman had repetitive superficial skin peeling and ensuing erythematous changes in the sites since infancy. Her parents had a consanguineous marriage, and she was the only individual affected in her family tree. The erythematous changes seemed to worsen in the summer. Histologically, hyperkeratosis and splitting of the epidermis within the stratum corneum was noted, and electron microscopy revealed shedding of corneal cells in the horny layer and normal-looking corneodesmosomes. Gene analysis revealed a homozygous missense mutation at c.1358G >A in CDSN. Electron microscopic examination of the length and number of corneodesmosomes revealed statistically significant shortness and sparsity in the affected individual (mean \pm SD $386.2 \pm 149.5 \mathrm{~nm}$ ) compared with that of an age- and site-matched control $(406.6 \pm 182.3 \mathrm{~nm})$. We speculate that this size shrinkage of corneodesmosomes might be the result of a missense mutation of CDSN and that this could be one of the factors contributing to the pathological process of skin peeling.
\end{abstract}


Kawakami et al.: A Case of Inflammatory Generalized Type of Peeling Skin Syndrome Possibly Caused by a Homozygous Missense Mutation of CDSN

\section{Introduction}

Peeling skin syndrome (PSS) is a rare autosomal recessive cornification disorder that is both clinically and genetically heterogeneous. PSS is characterized by shedding of the stratum corneum upon birth or in early infancy [1] and can be classified into two subtypes: acral PSS (peeling skin confined to the acral surface) and generalized PSS (systemic peeling). A mutation in transglutaminase 5 was identified as giving rise to acral PSS [2, 3]. The generalized type of PSS is further subclassified into non-inflammatory PSS (type A PSS) manifesting year-round peeling of the stratum corneum without inflammatory change, and inflammatory PSS (type B PSS) showing inflammatory changes resembling mild congenital ichthyosiform erythroderma. Type A PSS has been shown to have its genetic basis in the CHST8 gene encoding a Golgi transmembrane N-acetylgalactosamine-4-0-sulfotransferase (GALNAC4-ST1) in 2012 [4]. Type B PSS is known to be associated with mutations in several loci of corneodesmosin, a specific constituent protein of corneodesmosomes, in all of which the mode of mutation reported so far is exclusively homozygous nonsense, leading either to a truncated corneodesmosin protein or to messenger RNA transcript decay [5-8]. We report a case of type B PSS possibly caused by a homozygous missense mutation of CDSN.

\section{Case Report}

The patient was a 54-year-old Japanese woman who had experienced repetitive superficial skin peeling over the entire body and ensuing erythematous color changes at the sites since infancy. She did not have any children and was an offspring of a consanguineous marriage, but neither her four siblings nor anyone in her family tree reported any of the symptoms experienced by the patient. She claimed that the erythematous change worsened in the summer compared to the winter. Shedding of the skin was more prominent on the palms, forearms and intertriginous areas than on the torso (fig. 1). Hair shaft analysis did not show trichorrhexis invaginata.

A skin biopsy specimen was taken from the site of peeling on her right upper arm. Hematoxylin and eosin (H\&E) staining revealed basket weave-type hyperkeratosis with half of the stratum corneum in the right upper portion of the image seemingly sloughed off (fig. 2). The figure shows the original stratum corneum with the residual half above it and the area left of the center missing the upper corneal sheet. In the upper dermis, perivascular mononuclear infiltrate and mild dilation of capillaries were observed. Electron microscopy (fig. 3) showed splitting of the corneocyte rows. However, whether this change stemmed from a pathological cause or was an artefact is not clear. Corneodesmosomes were found between the corneocytes, and recognizable abnormalities in the density and morphology of corneodesmosomes could not be detected by viewing the images. The keratin aggregate pattern within the corneocytes and in the cornified envelope appeared normal.

Peripheral blood was collected for genetic analysis with the patient's consent. Biological materials could not be obtained from the patient's family members. As a result, a homozygous missense mutation was revealed at nucleotide position 1358 substituting adenine for guanine (c.1358G>A) in the CDSN gene, resulting in an exchange of the amino acid serine for asparagine (p.S453N). This mutation was not detected amongst the 100 healthy Japanese controls.

In order to determine whether there was any structural abnormality in our electron microscopic findings, we measured the length and number of corneodesmosomes per unit length both in our patient and in an age- and site-matched control. The corneodesmosomes 
Kawakami et al.: A Case of Inflammatory Generalized Type of Peeling Skin Syndrome

measured were confined to the second corneocyte layer above the stratum granulosum and carefully chosen from those that were horizontal to the stratum corneum. The results revealed a statistically significant shortening of corneodesmosomes in our case (mean \pm SD $386.2 \pm 149.5 \mathrm{~nm})$ compared with the healthy control $(446.3 \pm 185.8 \mathrm{~nm})$, as well as a lower corneodesmosome density in the affected individual $\left(0.860 \pm 0.233 \mu \mathrm{m}^{-1}\right)$ than in the control $(1.309 \pm 0.413)$ (table 1).

\section{Discussion}

The corneodesmosome, a modified form of desmosome found in the stratum corneum and inner root sheath of the hair follicles, differs ultrastructurally from the desmosome and is shown to play an important role in intercellular adhesion between corneocytes in the cornified cell layer [9]. The extracellular part of the corneodesmosome is mainly composed of corneodesmosin, desmoglein 1 and desmocollin 1 [10], which are together incorporated into the normal desquamation process as they lose their adhesive properties from gradual cleavage by enzymes known as kallikrein-related peptidases and cathepsins in the stratum corneum. Secreted from the lamellar granules of keratinocytes between the granular layer and the corneal layer, corneodesmosin, a 52-56-kDa glycoprotein, is incorporated into a structure known as the desmoglea before they are transformed into corneodesmosomes in the process of cornification.

A series of reports has established that type B PSS is caused by deleterious mutations in the CDSN gene encoding corneodesmosin. A comparison of all reported loci of mutation, their histological findings and serum IgE levels (table 2) revealed that the homozygous missense mutation in the present case lay very far downstream from the previously known mutations, which were all homozygous nonsense. Most of the type B PSS cases presented remarkably high serum IgE compared with the present case, suggesting that the relative mildness of symptoms and the relatively lower serum IgE level may correlate with less barrier function deficiency in our patient.

The biopsy specimen in all of the reported cases demonstrated compact hyperkeratosis and detachment of the corneal sheet immediately above the stratum granulosum as shown by H\&E staining (table 2). In contrast, our case showed neither stuck hyperkeratosis nor subcorneal cleavage, and the image was rather characterized by basket weave-type hyperkeratosis and possible shedding of the corneal sheet amid the stratum corneum. This histopathological aberrance from previously reported cases might be explained by the presence of solid corneodesmosomes detected by electron microscopy in our patient. Although it is difficult to determine to what extent our missense mutation affected the adhesive property of the corneodesmosomes, it is possible that they may have remained partly functional, though not sufficiently so to execute the normal desquamation process.

Measurements of the corneodesmosomes by electron microscopy revealed shrinkage and sparsity in our case (table 1), but the structure itself seemed intact, as evidenced by the homogeneously electron-dense structure. Only one report of type B PSS, in which a homozygous nonsense mutation was identified at the upstream locus of $C D S N$, examined the electron microscopic view of corneodesmosomes and revealed hardly visible corneodesmosomes immediately above the stratum granulosum [5]. This might explain how the desmoglea does not phenotypically form and become visible without non-truncated corneodesmosin, and furthermore how the downstream locus with homozygous missense mutation in our case might affect morphologically normal-looking corneodesmosome formation. 
Kawakami et al.: A Case of Inflammatory Generalized Type of Peeling Skin Syndrome Possibly Caused by a Homozygous Missense Mutation of CDSN

We have reported a case of type B PSS possibly caused by a homozygous missense mutation of $C D S N$. Further cumulative, analytic studies of CDSN mutations will undoubtedly elucidate the genotype-phenotype correlation as well as the detailed function of corneodesmosin.

\section{References}

1 Levy SB, Goldsmith LA: The peeling skin syndrome. J Am Acad Dermatol 1982;7:606-613.

2 Cassidy AJ, van Steensel MA, Steijlen PM, van Geel M, van der Velden J, Morley SM, Terrinoni A, Melino G, Candi E, McLean WH: A homozygous missense mutation in TGM5 abolishes epidermal transglutaminase 5 activity and causes acral peeling skin syndrome. Am J Hum Genet 2005;77:909-917.

3 Kharfi M, El Fekih N, Ammar D, Jaafoura H, Schwonbeck S, van Steensel MA, Fazaa B, Kamoun MR, Fischer J: A missense mutation in TGM5 causes acral peeling skin syndrome in a Tunisian family. J Invest Dermatol 2009;129:2512-2515.

4 Cabral RM, Kurban M, Wajid M, Shimomura Y, Petukhova L, Christiano AM: Whole-exome sequencing in a single proband reveals a mutation in the CHST8 gene in autosomal recessive peeling skin syndrome. Genomics 2012;99:202-208.

5 Oji V, Eckl KM, Aufenvenne K, Nätebus M, Tarinski T, Ackermann K, Seller N, Metze D, Nürnberg G, Fölster-Holst R, Schäfer-Korting M, Hausser I, Traupe H, Hennies HC: Loss of corneodesmosin leads to severe skin barrier defect, pruritus, and atopy: unraveling the peeling skin disease. Am J Hum Genet 2010;87:274-281.

6 Israeli S, Zamir H, Sarig O, Bergman R, Sprecher E: Inflammatory peeling skin syndrome caused by a mutation in CDSN encoding corneodesmosin. J Invest Dermatol 2011;131:779-781.

7 Telem DF, Israeli S, Sarig 0, Sprecher E: Inflammatory peeling skin syndrome caused a novel mutation in CDSN. Arch Dermatol Res 2012;304:251-255.

8 Mazereeuw-Hautier J, Leclerc EA, Simon M, Serre G, Jonca N: A novel mutation in CDSN causes peeling skin disease in a patient from Morocco. Br J Dermatol 2011;165:1152-1155.

9 Chapman SJ, Walsh A: Desmosomes, corneosomes and desquamation. An ultrastructural study of adult pig epidermis. Arch Dermatol Res 1990;282:304-310.

10 Caubet C, Jonca N, Brattsand M, Guerrin M, Bernard D, Schmidt R, Egelrud T, Simon M, Serre G: Degradation of corneodesmosome proteins by two serine proteases of the kallikrein family, SCTE/KLK5/hK5 and SCCE/KLK7/hK7. J Invest Dermatol 2004;122:1235-1244.

Table 1. Comparison of the length and number of corneodesmosomes per unit length between our case and an age- and site-matched control

\begin{tabular}{llll}
\hline & Our case & Healthy control & F value \\
\hline Length of corneodesmosomes, $\mathrm{nm}($ mean \pm SD) & $386.2 \pm 149.5$ & $446.3 \pm 185.8$ & $0.015(<0.05)$ \\
Density of corneodesmosomes, $\mu \mathrm{m}^{-1}($ mean \pm SD) & $0.860 \pm 0.233$ & $1.309 \pm 0.413$ & $0.038(<0.05)$ \\
\hline
\end{tabular}


Kawakami et al.: A Case of Inflammatory Generalized Type of Peeling Skin Syndrome Possibly Caused by a Homozygous Missense Mutation of CDSN

Table 2. Comparison between previously reported cases and our case in terms of mutation loci, mode of mRNA translational errors, histopathological features and serum IgE level

\begin{tabular}{lllll}
\hline Reference & $\begin{array}{l}\text { Genetic mutations } \\
\text { (affected amino acids) }\end{array}$ & $\begin{array}{l}\text { Mode of mRNA translational } \\
\text { errors }\end{array}$ & H\&E staining & $\begin{array}{l}\text { Serum IgE, } \\
\text { IU/ml }\end{array}$ \\
\hline $\begin{array}{l}\text { Oji et al. [5], } \\
2010\end{array}$ & c.175A>T (p.K59X) & homozygous nonsense & $\begin{array}{l}\text { compact hyperkeratosis; } \\
\text { detachment directly above } \\
\text { the striatum granulosum }\end{array}$ & $>2,000$ \\
\hline $\begin{array}{l}\text { Telem et al. [7], } \\
2012\end{array}$ & $\begin{array}{l}\text { c.164_167dup GCCT } \\
\text { (p.T57PfsX6) }\end{array}$ & $\begin{array}{l}\text { homozygous duplication } \\
\rightarrow \text { stop codon }\end{array}$ & $\begin{array}{l}\text { compact hyperkeratosis; } \\
\text { subcorneal separation }\end{array}$ & unknown \\
\hline $\begin{array}{l}\text { Israeli et al. [6], } \\
2011\end{array}$ & c.746delG (p.G249VfsX40) & $\begin{array}{l}\text { homozygous single nucleotide } \\
\text { deletion } \rightarrow \text { stop codon }\end{array}$ & $\begin{array}{l}\text { compact hyperkeratosis; } \\
\text { intracorneal and subcorneal } \\
\text { separation }\end{array}$ & 30,375 \\
\hline $\begin{array}{l}\text { Mazereeuw et al. } \\
\text { [8], 2011 }\end{array}$ & c.754delC (p.P252LfsX37) & $\begin{array}{l}\text { homozygous single nucleotide } \\
\text { deletion } \rightarrow \text { stop codon }\end{array}$ & $\begin{array}{l}\text { compact hyperkeratosis; } \\
\text { subcorneal separation }\end{array}$ & 23,000 \\
\hline Our case & c.1358G>A (p.S453N) & homozygous missense & $\begin{array}{l}\text { basket weave-type } \\
\text { hyperkeratosis; intracorneal }\end{array}$ & 369 \\
& & & separation & \\
\hline
\end{tabular}


Kawakami et al.: A Case of Inflammatory Generalized Type of Peeling Skin Syndrome Possibly Caused by a Homozygous Missense Mutation of CDSN
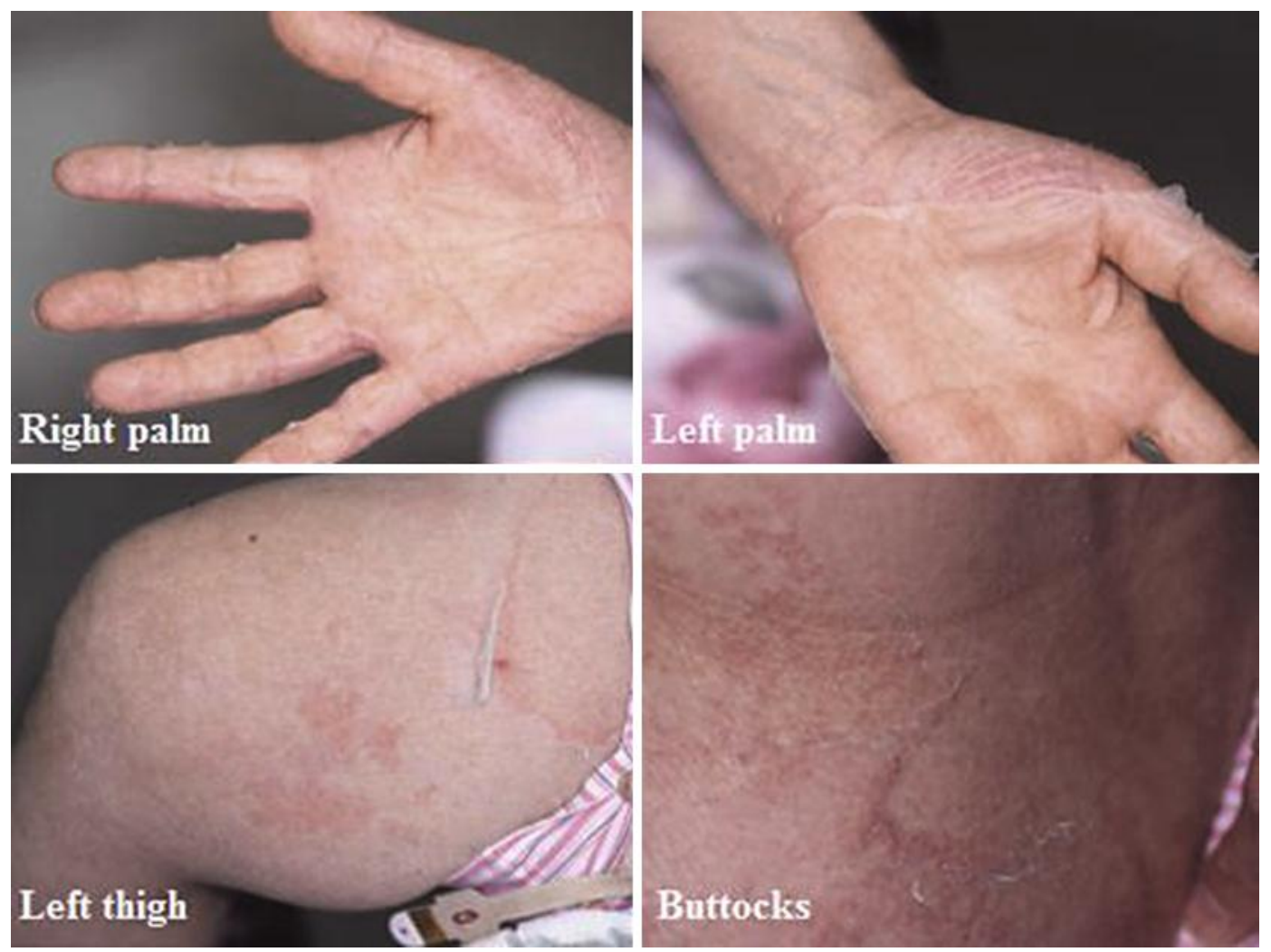

Fig. 1. Peeling of the skin was noted on both palms, thighs and buttocks. Erythematous color changes at the site of peeling were observed. Shedding of the skin was observed more prominently on the palms, forearms and intertriginous areas than on the torso. 
Kawakami et al.: A Case of Inflammatory Generalized Type of Peeling Skin Syndrome Possibly Caused by a Homozygous Missense Mutation of CDSN

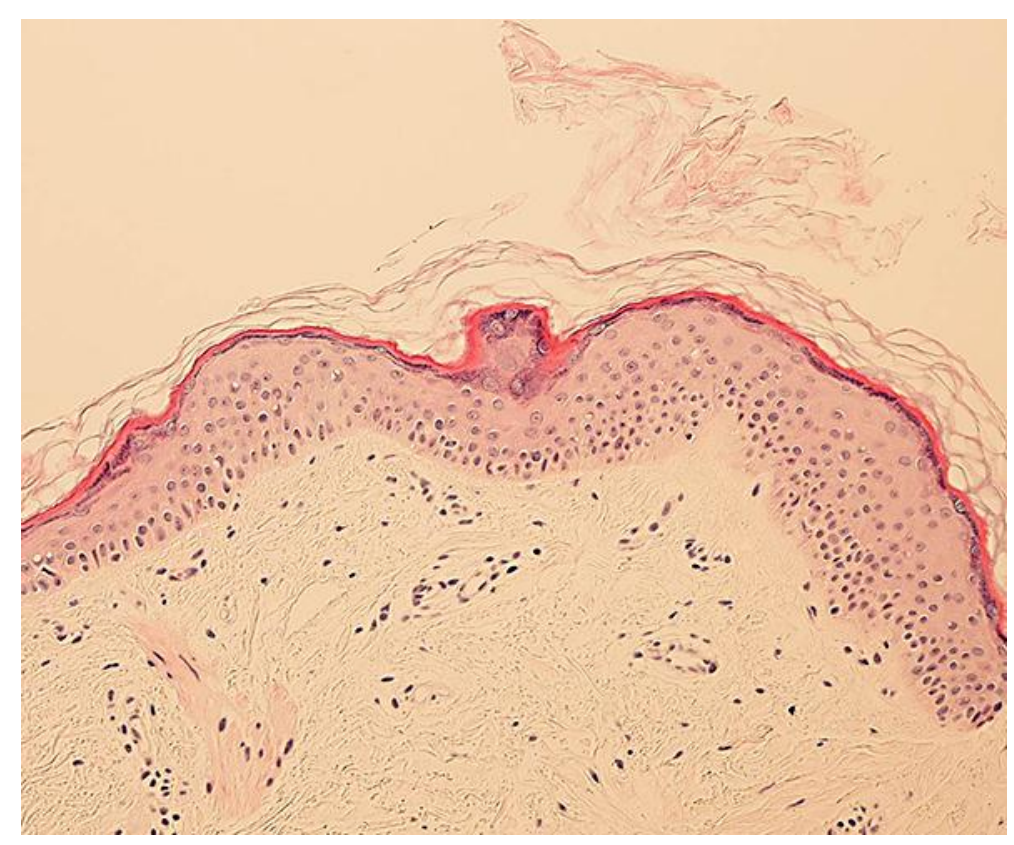

Fig. 2. Basket weave-type hyperkeratosis with half of the stratum corneum in the right upper portion of the image seemingly sloughed off. The figure shows the original stratum corneum with the residual half above it and the area left of the center missing the upper corneal sheet.

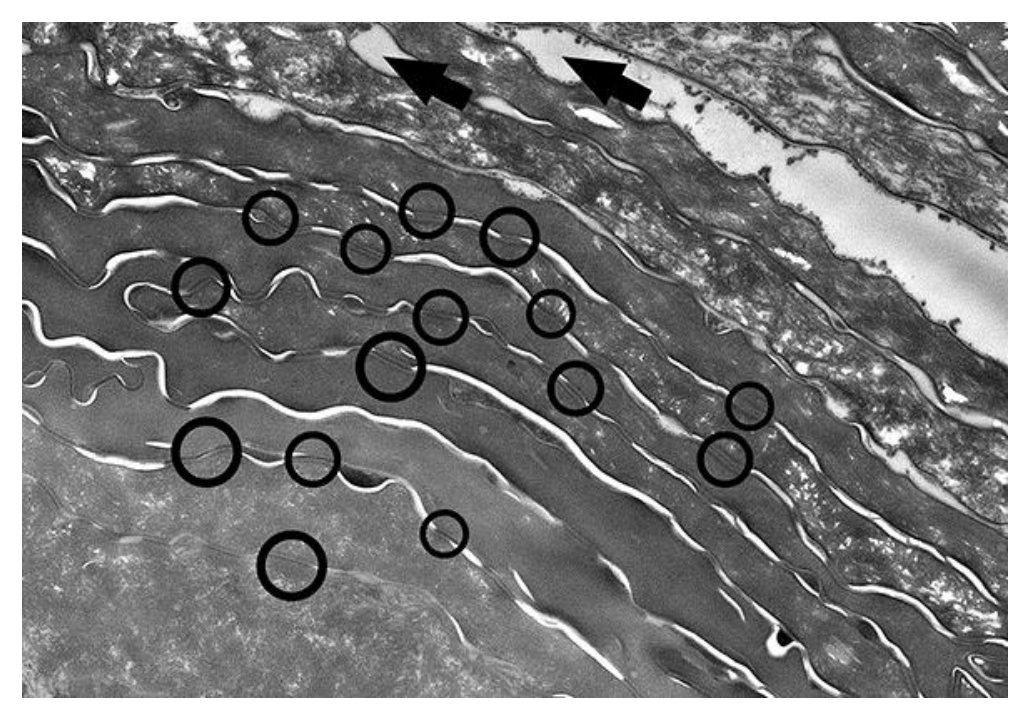

Fig. 3. Electron microscopy showed split formation (arrows) between the corneocyte rows. However, whether this change stemmed from a pathological cause or was an artefact is not clear. As shown by the circles, corneodesmosomes were found between the corneocytes, and abnormalities in the density and morphology of the corneodesmosomes were not obvious. 